\title{
Paraneoplastic encephalomyelitis: Is it an oropharyngeal or a lung cancer complication?
}

\author{
MARÍA SERENO MOYANO ${ }^{1}$, GERARDO GUTIÉRREZ-GUTIÉRREZ ${ }^{2}$, CÉSAR GÓMEZ-RAPOSO ${ }^{1}$, \\ MIRIAM LÓPEZ GÓMEZ ${ }^{1}$, JOAQUÍN OJEDA ${ }^{2}$, AMBROSIO MIRALLES ${ }^{2}$ and ENRIQUE CASADO-SÁENZ ${ }^{1}$ \\ Departments of ${ }^{1}$ Oncology, and ${ }^{2}$ Neurology, Infanta Sofía Hospital, San Sebastián de los Reyes, Madrid, Spain
}

Received September 30, 2010; Accepted November 12, 2010

DOI: $10.3892 / 01.2010 .224$

\begin{abstract}
This case report describes a patient with a locally advanced oropharyngeal cancer with a simultaneous paraneoplastic encephalomyelitis. To the best of our knowledge, a paraneoplastic neurological syndrome is a rare complication in head and neck cancer, and has previously not been reported in the literature. One year later, following initial treatment, a small cell lung cancer developed, a tumor frequently associated with this type of paraneoplastic syndrome. The dilemma, therefore, is whether this paraneoplastic symdrome was a secondary complication of the tonsilar concurrent cancer or a metachronous paraneoplastic syndrome prior to small cell lung cancer.
\end{abstract}

\section{Introduction}

Cancer of the head and neck (HNC) refers to a various types of cancer, predominantly squamous cell carcinomas, arising from a variety of sites and grouped together under this category, including oropharyngeal cancer. Worldwide, cancers of the oropharynx and hypopharynx account for an estimated 123,000 new cases per year, with an estimated mortality of 79,000 deaths (1). Surgery and/or radiotherapy are the standard treatment for oropharyngeal cancer, and these modalities are frequently complicated by the suboptimal control of locoregional disease and significant long-term functional deficits (1).

Paraneoplastic neurological syndromes (PNS) are defined as remote effects of cancer that are not caused by the tumor and its metastasis, nor by infection, ischemia, toxicity of cancer therapy, coagulopathy or metabolic disruptions (2). In the majority of patients, neurological disorders develop before the cancer becomes clinically overt. In the last two decades, the finding that certain PNS are associated with antibodies directed against tumoral antigens expressed by the

Correspondence to: Dr María Sereno Moyano, Oncology Department, Infanta Sofía Hospital, c/Julio Rey Pastor n6, Portal 6, 28702 San Sebastián de los Reyes, Madrid, Spain

E-mail: mariasereno75@gmail.com

Key words: oropharyngeal cancer, paraneoplastic encephalomyelitis tumor and the nervous system suggests that these disorders are immune-mediated. Numerous onconeural antibodies have been described, but less than $50 \%$ of patients with PNS harbor these types of proteins.

Therefore, the absence of paraneoplastic antibodies does not exclude the diagnosis of PNS. The main neurological syndromes associated with paraneoplastic origin include limbic encephalitis, subacute cerebellar ataxia, opsoclonus-myoclonus, retinopathies, chronic gastrointestinal pseudoobstruction, sensory neuronopathy, Lambert-Eaton myasthenic syndrome and encephalomyelitis (3).

The majority of tumors associated with PNS are small cell lung cancer (SCLC), ovarian cancer and hematological diseases, mainly lymphomas. PNS in HNC is extremely rare. No publications are currently available on the association between squamous oropharyngeal cancer and neurological paraneoplastic disease (4).

Recently, we encountered a patient with a locally advanced oropharyngeal cancer who simultaneously developed a PNS-type encephalomyelitis. To the best of our knowledge, a PNS in a patient with HNC has not previously been reported.

\section{Case report}

A 58-year-old male was admitted to the emergency room. Two to three days earlier, he had experienced weakness in his left leg resulting in difficulty in walking, which the day prior to admission had progressed to affect the left arm and the right part of the face. No prior medical conditions or surgeries were noted. The patient was a social drinker and he usually smoked one pack of cigarettes per day.

A number of weeks prior to admission, the patient experienced hypoesthesia to cold and hot stimuli in his right leg. He also reported that he occasionally suffered pain in the throat and noted blood in his spittle.

A general physical examination showed no abnormal findings. However, a neurological examination revealed right facial palsy, mild brachiocrural left hemiparesis with increased tendon reflexes and a Babinski response on the left side. The laboratory values were within normal limits. A chest X-ray did not show any relevant findings. The brain and body CT was normal. In the ear, nose and throat (ENT) examination, a small lesion $(<1 \mathrm{~cm})$ in the right tonsilar bed was found and a biopsy sample was removed. Pathological examination 
of the sample was consistent with squamous cell carcinoma. The MRI and CT (Fig. 1) of the neck showed a heterogeneous mass located on the right tonsilar area involving the pterygoideus medial muscle and the pre-styloid parapharingeus space. This mass grew inside the back wall of the oropharynx, and presumably involved the long neck muscle on the right side. Certain cervical lymph nodes were present in the IIA and IIB spaces, the largest diameter being $\sim 2.5 \mathrm{~cm}$. According to the endoscopic and radiological findings, the TNM classification was T4N2bM0 (IVA).

The patient's neurological symptoms became progressively worse. The brain and spine MRI showed a small lesion in the right protuberance with subtle changes in the DW sequence that were enhanced following contrast infusion, and another lesion in the cervical spinal cord that may explain the neurological findings (Fig. 2). In addition, a number of small subcortical lesions were found (Fig. 3). The CSF exam revealed a Tibbling index of 1.1, protein concentration $66 \mathrm{mg} / \mathrm{dl}$, and normal counts of glucose and cells. A microbiological examination did not reveal any signs of infection and three cytologies performed for malignant cells were negative. Anti-nuclear antibodies, endothelial cytotoxic activity and serum tests (vitamin B12, $\mathrm{IgG}$, tumoral markers and thyroid hormones) were normal. The microbiological examination included serology for RPR and THPA. Hepatitis B virus, human immunodeficiency virus, toxoplasmosis, citomegalovirus, herpes-simplex virus type 6 and Varicela zoster virus, Epstein-barr virus, mycoplasma pneumonia and Borrelia burgdorferi were normal. Finally,

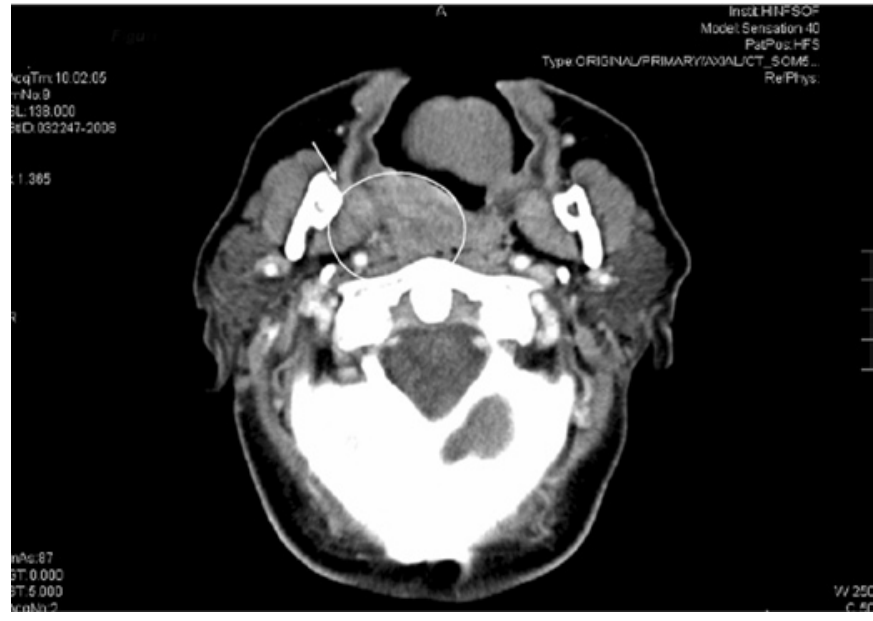

Figure 1. The CT showed a heterogeneous mass located on the right tonsilar area involving pterygoideus medial muscle and the pre-styloid parapharingeus space. This mass grew inside the back wall of the oropharynx, and presumably involved the right long neck muscle. Certain cervical lymph nodes were present in the IIA and IIB spaces, the largest diameter being $\sim 2.5 \mathrm{~cm}$.

since paraneoplastic encephalomyelitis is occasionally associated with certain types of cancer, a number of onconeural antibodies, including anti-Hu, anti-Jo, anti-Ri, anti-MA-1 and anti-CV2, were examined to eliminate the possibility of a neurological paraneoplastic syndrome. Presentation of the antibodies was not demonstrated; however PNS could not be

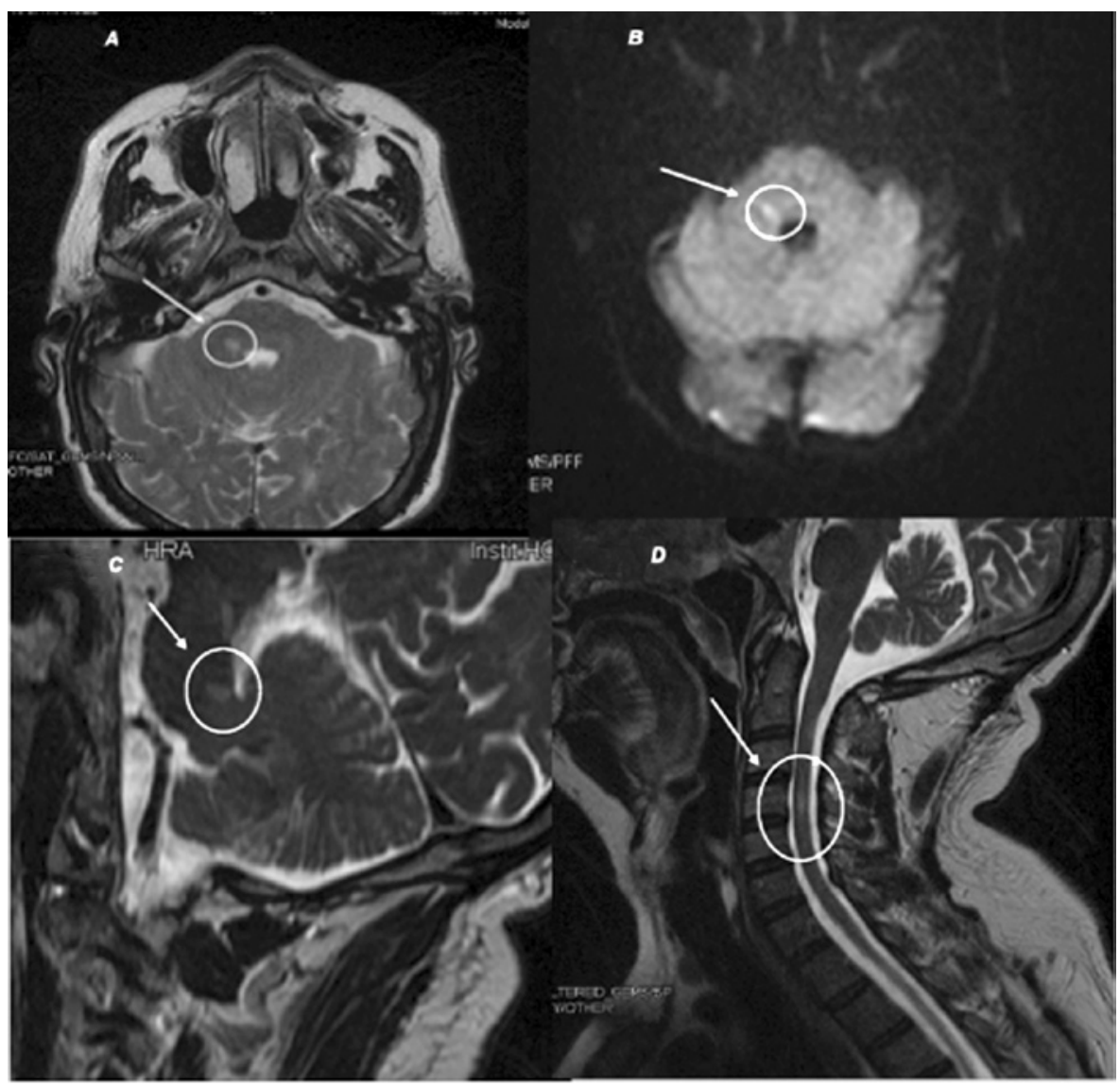

Figure 2. MRI showed a (A) small lesion in the right protuberance (arrow) with (B and C) subtle changes in the DW sequence that were enhanced following contrast infusion, and (D) another lesion in the cervical spinal cord. 


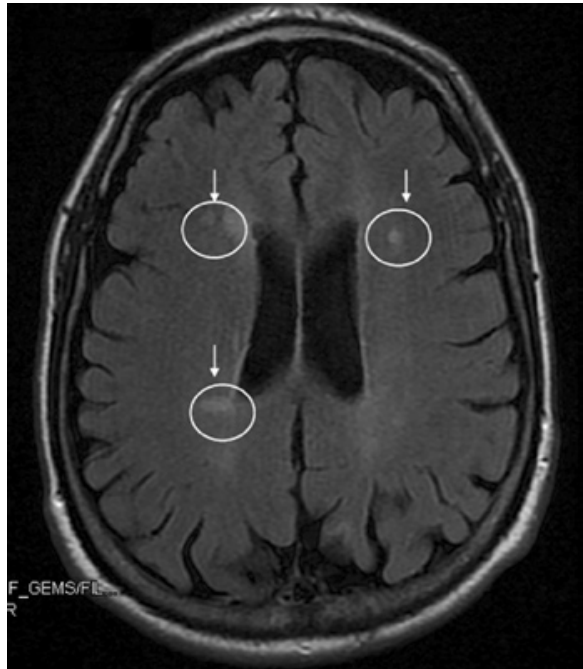

Figure 3. Small subcortical lesions.

excluded according to the accepted criteria. Corticoid treatment was initiated with mild and transitory improvement of neurological symptoms, which is consistent with our initial hypothesis.

This patient received three courses of cisplatin, taxotere and 5-fluorouracil (5). The radiological and ENT examinations showed complete response in that no residual tumor was found in the biopsy sample. Lymph nodes were not observed when the MRI was performed. However, the neurological symptoms persisted, with the addition of numbness of feet. Cisplatin-related neuropathy was confirmed by an electromyogram study. Subsequently, administration of this neurotoxic drug was suspended and an anti-EGFR monoclonal antibody $\left(\right.$ Erbitux $\left.^{\circledR}\right)$, without neurological side effects, was added.

The patient then underwent a chemoradiation treatment (tomotherapy and Erbitux, concomitantly), with cutaneous toxicity grade II. One month later, the MRI and endoscopic examination revealed a pathologically complete response, resulting in the patient undergoing surgery (bilateral lymph nodes resection). The pathological examination did not reveal metastasis in the nodes that were removed. Follow-up was conducted every 2 months and comprised an ENT examination, blood test and alternating image test (MRI and neck CT). No evidence of relapse has been detected thus far. However, the neurological symptoms became worse, even after cisplatin suppression. The patient was unable to walk independently and attended the hospital in a wheelchair. Additionally, he reported urinary retention 2 weeks prior to the last appointment.

Corticoides therapy did not improve the neurological symptoms and the MRI showed an increase in lesion size. It was concluded that our patient may have a PNS (type, encephalomyelitis) associated with a HNC. He presented with a neurological syndrome that is not attributable to the toxicity of cancer therapy, cerebrovascular disease, coagulopathy, infection or toxic and metabolic causes. The neurological diagnosis included the criteria required to define a possible PNS, i.e., non-classical neurological syndrome (encephalomyelitis) without onconeural antibodies, and the cancer presents within 2 years of diagnosis.

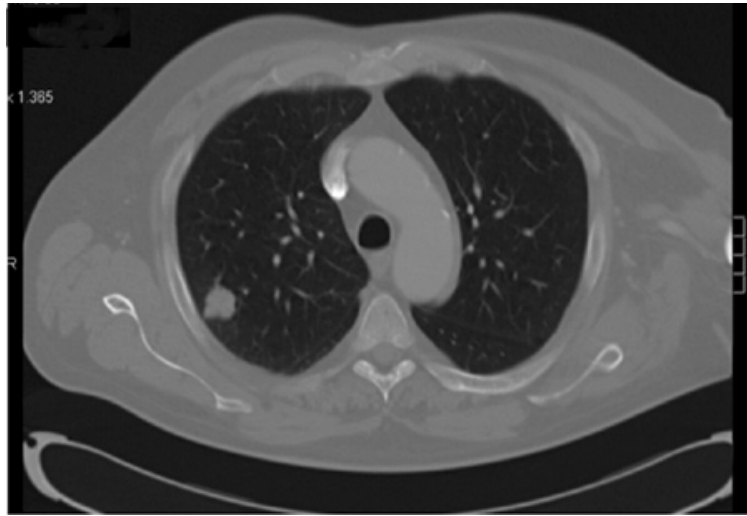

Figure 4. Chest CT scan; a 3-cm nodule in the upper right lobe.

One year later, a chest CT scan showed a 3-cm nodule in the upper right lobe. A biopsy was performed, the finding of which were consistent with a SCLC. The work-up did not show metastasis in any other location. Chemotherapy and radiation treatment were commenced in another hospital due to relocation. A partial response to the treatment was noted (Fig. 4).

Thus, we have presented the case of a patient, with a locally advanced tonsilar carcinoma, who underwent chemoradiation with a complete response, as well as paraneoplastic encephalomyelitis associated with peripheral neuropathy secondary to cisplatin administration, is presented. This is potentially the first case of a possible PNS associated with a HNC or a complication of a further SCLC reported in the literature.

\section{Discussion}

PNS is a rare disease occurring in less than $0.01 \%$ of patients with cancer (1). The current criteria for PNS were reviewed by an international panel of neurologists (2). Paraneoplastic encephalomyelitis is characterized by the involvement of one or more areas, such as the hippocampus, lower brainstem, spinal cord or dorsal root ganglia. In $75 \%$ of patients, the underlying neoplasm is a SCLC and a majority of patients present anti-Hu, anti-CV2 or anti-amphiphysin antibodies.

The field cancerization hypothesis is a key concept related to the natural history of HNC. This term describes the diffuse epithelial injury throughout the head and neck, lungs and esophagus that results from chronic exposure to carcinogens (3). This lesion may be responsible for the development of a second tumor in the lungs or oesophagus in the damaged areas. Therefore, our patient may develop a second tumor in the lungs or in the oesophagus as a consequence of this process.

According to the previous criteria defining PNS, the neurological syndrome that we have presented may be associated with an underlying lung cancer instead of a HNC. As previously mentioned, lung cancer is more frequently associated with PNS, in particular, SCLC. Therefore, the presence of a PNS in our patient may potentially be explained by the fact that either the second tumor is a definite PNS associated with a HNC or it is a possible PNS associated with future lung cancer in a patient with high risk, according to the 'field cancerization' hypothesis. In the case that we consider the tumor to be a paraneoplastic syndrome associated with HNC, 
this would be the first such reported case. HNC outcome did not show a local or distant recurrence during the 1 year of follow-up.

One year later, a second primary tumor was noted in the lung. This tumor was a SCLC, which is frequently associated with paraneoplastic syndrome tumors. Therefore, this paraneoplastic syndrome may be explained as a complication prior to the appearance of SCLC.

In conclusion, we examined a patient who concurrently presented a neurological syndrome of encephalomyelitis and a tonsilar mass with a biopsy consistent with carcinoma. According to the clinical and radiological findings, as well as the clinical outcome, we conclude that this mass is a possible PNS. However, we cannot assert whether it is a PNS associated with a HNC or a possible PNS that resulted in the development of a second tumor in the lungs as a result of field cancerization.

\section{Acknowledgements}

We thank Dr Dominguez from the Pathology Department in our hospital for her expert contribution.

\section{References}

1. Licitra L, Bernier J, Grandi C, et al: Cancer of the oropharynx. Crit Rev Oncol Hematol 41: 107-122, 2001.

2. Graus F, Delattre JY, Antoine JC, et al: Recommended diagnostic criteria for paraneoplastic neurological syndromes. J Neurol Neurosurg Psychiatry 75: 1135-1140, 2004.

3. Honnorat J and Antoine JC: Paraneoplastic neurological syndromes. Orphanet J Rare Dis 22: 1-8, 2007.

4. Scheid R, Honnorat J, Delmont E, et al: Neurological disorders in patients with small cell lung cancer. Cancer 60: 2275-2283, 1987.

5. Vermorken JB, Remenar E, van Herpen C, et al: Cisplatin, fluorouracil, and docetaxel in unresectable head and neck cancer. N Engl J Med 357: 1704-1715, 2007. 